\title{
La incorporación de la mujer en la cirugía chilena*
}

\author{
Drs. RICARDO ESPINOZA G. ${ }^{1}$, CAMILA ONETTO C. ${ }^{1}$, \\ JUAN JOSÉ LOMBARDI A. ${ }^{1}$, Int. JUAN PABLO ESPINOZA G. ${ }^{1}$ \\ 1 Departamento de Cirugía. Facultad de Medicina. Universidad de Los Andes. \\ Santiago, Chile.
}

\begin{abstract}
The incorporation of women in Chilean surgery

It has been observed an increasing number of women studying medicine. However, the incorporation of women to surgery remains low. In Chile we do not have information on this topic. Our goal was to collect information to provide the figures and trends in this regard. Information was obtained from the Society of Surgeons of Chile (SCCH), the Chilean Chapter of the American College of Surgeons (CCh CAC), the Autonomous National Commission of Medical Specialties (CONACEM) and the Superintendent of Health of Chile (SS), until June 2011. The SCCH has 854 partners and 64 were women (7.5\%). The Chilean Chapter of the CCh CAC has 162 members and 4 women (2.5\%). At CONACEM, 1.070 persons have been certified as surgeons, 80 are women (7.5\%). In the register of SS there are 1.177 surgeons, $8.4 \%$ female. In 1959 the first women joined SCCH. Since then, the number has been progressive, growing mainly in the last two decades to reach the $7.5 \%$ now. Nevertheless, in all consulted sources, the percentage of women in surgical practice, still less than $10 \%$. It seems that woman is trying to increase participation in the surgical field. However, the "surgeon's lifestyle" collides with compatibility between work and family life. We believe there is room for qualitative research in this field, to better understand motivations and challenges of women in order to access the surgical world nowadays.
\end{abstract}

Key words: Women, surgery.

\section{Resumen}

Se ha observado un aumento del número de mujeres que estudia medicina. No obstante, la incorporación de la mujer a la cirugía sigue siendo baja. En Chile no conocemos de información sobre este tema. Nuestro objetivo fue recopilar información que proporcionara cifras y tendencias en este sentido. Se obtuvo información de la Sociedad de Cirujanos de Chile (SCCh), del Capítulo Chileno del Colegio Americano de Cirujanos (CCh CAC), de la Comisión Nacional Autónoma de Especialidades Médicas (CONACEM) y de la Superintendencia de Salud de Chile (SS), hasta junio de 2011. La SCCh tiene 854 socios y 64 corresponden a mujeres (7,5\%). El Capítulo Chileno del CAC tiene 162 miembros y 4 son mujeres (2,5\%). Ante CONACEM se han certificado 1.070 cirujanos, de los cuales 80 son mujeres (7,5\%). En el registro de la SS hay 1.177 ci-

*Recibido el 23 de enero de 2012 y aceptado para publicación el 12 de mayo de 2012.

Los autores no declaran conflictos de interés.

Correspondencia: Dr. Ricardo Espinoza G.

Av. San Carlos de Apoquindo 2200, Santiago, Chile.

respinoza@clinicauandes.cl 
rujanos, 8,4\% de sexo femenino. El año 1959 se incorporó la primera mujer a la SCCh. Desde ahí, el número ha sido progresivo, aumentando principalmente en las dos décadas recientes hasta alcanzar el 7,5\% actual. No obstante este aumento, en todas las fuentes consultadas el porcentaje de mujeres en práctica quirúrgica es inferior al 10\%. Pareciera ser que la mujer intenta realmente una mayor participación en el campo quirúrgico. Sin embargo, el "estilo de vida del cirujano", choca con una compatibilización mayor entre vida laboral y familiar. Creemos que hay espacio para investigaciones cualitativas en este campo, que reflejen mejor las motivaciones y dificultades de la mujer por acceder al mundo quirúrgico en nuestro medio.

Palabras clave: Mujer, cirugía.

\section{Introducción}

En las últimas décadas se ha venido observando un marcado aumento del número de mujeres que se incorpora a los estudios de medicina. En los Estados Unidos de Norteamérica prácticamente el 50\% de los alumnos de pregrado es de sexo femenino ${ }^{1}$. Para los años 1999-2000, Novielli² ${ }^{2}$ señala que esta cifra correspondió exactamente a un 45,6\%. En una reciente publicación de Argentina se indica que el 58,8\% de los graduados de medicina en ese país corresponde a mujeres ${ }^{3}$. Una publicación inglesa revela un $62 \%{ }^{2}$. En nuestro país, en el año 2010, de los 1.242 egresados, 587 eran mujeres (47,3\%). Sin embargo, la incorporación de la mujer en las diferentes especialidades muestra diferencias; específicamente la participación en el campo de la cirugía aún cuando también ha aumentado, sigue siendo baja. En términos generales se señala que las mujeres que ocupan posiciones académicas en los departamentos quirúrgicos varían entre un 10\% y un 14\%, y que sólo el $25 \%$ de los residentes de cirugía son mujeres ${ }^{1}$.

En Chile este análisis no ha sido hecho, de modo que no se dispone de forma sistematizada de algunos indicadores de la participación femenina en cirugía ni de la tendencia de la incorporación de la mujer al campo de la cirugía y sus especialidades. Nuestro objetivo fue recopilar información que proporcionara cifras y tendencias en este sentido.

Para obtener la información deseada recurrimos a los registros de la Sociedad de Cirujanos de Chile (SCCh), a los del Capítulo Chileno del Colegio Americano de Cirujanos (CChCAC), al registro de la Comisión Nacional Autónoma de Especialidades Médicas (CONACEM) y, finalmente a la nómina de prestadores individuales que mantiene la Superintendencia de Salud de Chile (SS). La Sociedad de Cirujanos fue fundada en 1949, por la fusión de la Sociedad de Cirugía de Chile (1922) y la Sociedad de Cirujanos de Hospital (1931). El Capítulo Chileno de Colegio Americano de Cirujanos data de 1952. CONACEM inició sus actividades en 1982 y la Superintendencia abrió la inscripción de especialistas en el año 2009. 2011.

Se consideraron los datos disponibles a junio de
En 1959 se incorporó la primera mujer cirujano a la SCCh y no se volvieron a registrar incorporaciones hasta la década de 1981-1990. Después, en los períodos 1991-2000 y 2001-2010 se produjo un significativo aumento de mujeres socias, como se observa en la Figura 1. En la actualidad, y hasta junio de 2011, la SCCh mantiene una nómina de 854 socios, de los cuales 64 corresponden a mujeres (7,5\%) (Figura 1).

El Capítulo Chileno del Colegio Americano de Cirujanos a la fecha tiene 162 miembros, de los cuales sólo 4 son mujeres (2,5\%), habiendo existido una quinta, que falleció.

En CONACEM se han certificado 1.070 cirujanos; 80 son mujeres $(7,5 \%)$ con un crecimiento que se observa en la Figura 2. En el registro de la Superintendencia de Salud existen 1.177 cirujanos certificados como tal, siendo el 8,4\% de sexo femenino $(\mathrm{n}=99)$. La totalidad de estos datos se aprecia en la Tabla 1, Figuras 2.

En Chile, en 1877 se autorizó el ingreso de mujeres a estudiar medicina, titulándose la Dra. Eloísa Díaz Insunza en $1886^{4}$. No conocemos quien fue la primera mujer que en Chile optó por la práctica quirúrgica, más, no fue sino hasta 1959 cuando se incorporó oficialmente la primera mujer cirujano a la Sociedad de Cirujanos de Chile. Tampoco existían programas universitarios de formación en la especialidad. Ello no descarta que anteriormente

Tabla 1. Mujeres Cirujanos en Chile

\begin{tabular}{|c|c|c|c|}
\hline \multirow[t]{2}{*}{ Registro } & \multirow{2}{*}{$\begin{array}{c}\text { Total socios } \\
\text { (n) }\end{array}$} & \multicolumn{2}{|c|}{ Mujeres socias } \\
\hline & & (n) & $(\%)$ \\
\hline CChCAC & 162 & 4 & 2,5 \\
\hline SCCh & 854 & 64 & 7,5 \\
\hline CONACEM & 1.070 & 80 & 7,5 \\
\hline SS & 1.177 & 99 & 8,4 \\
\hline
\end{tabular}

CChCAC: Capítulo Chileno Colegio Americano de Cirujanos. SCCh: Sociedad de Cirujanos de Chile. CONACEM: Comisión Nacional Autónoma de Especialidades Médicas. SS: Superintendencia de Salud. 

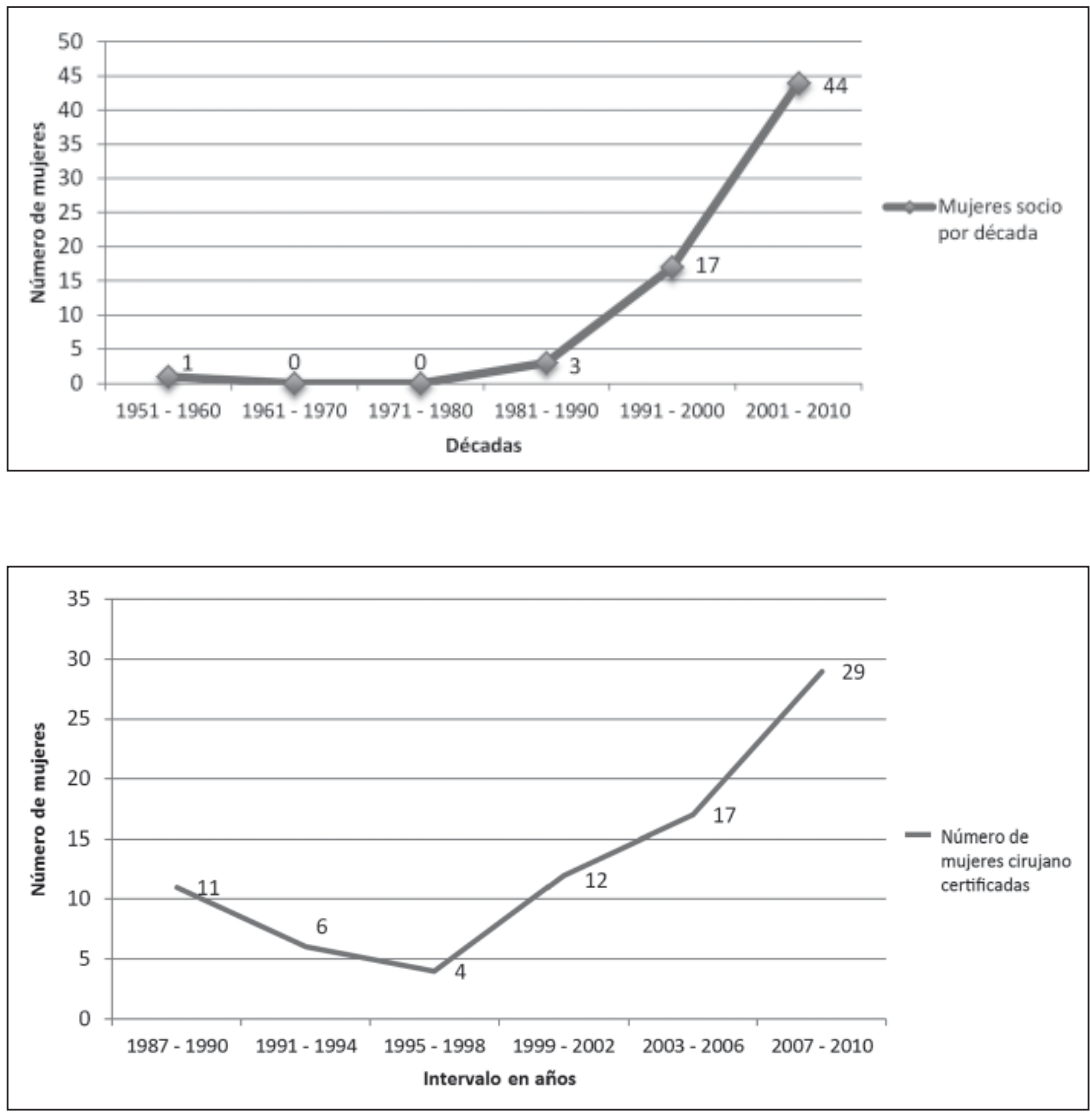

Figura 1. Mujeres pertenecientes a la Sociedad de Cirujanos de Chile.
Figura 2. Mujeres cirujano certificadas por CONACEM. hubiese otras mujeres dedicadas a la cirugía, considerando que, además, la incorporación societaria es voluntaria. Sin embargo, el año 1959 es cuando se reconoce oficialmente como cirujano a una mujer y es aceptada por sus pares como socia, y con todos los derechos. Desde ahí, el número ha sido progresivo, principalmente en las dos décadas recientes, aun cuando en cualquiera de las cuatro fuentes consultadas el porcentaje de mujeres en práctica quirúrgica es inferior al 10\%. Internacionalmente muchas investigaciones se han dirigido a estudiar esta baja representación de la mujer en el campo quirúrgico, llegando a hablarse de la triple barrera: son menos las mujeres que al ingresar a los estudios de medicina se interesan por la cirugía (6\% de mujeres versus $20 \%$ de hombres en un estudio y $18 \%$ y $50 \%$ en otro, respectivamente); es mayor la mayor proporción de mujeres que durante los estudios pierde el interés por esta especialidad (76\% mujeres versus $50 \%$ de los hombres) y, contrariamente, sólo un $6 \%$ de las mujeres que estudian medicina desarrollan interés por cirugía mientras cursan su pregrado, a diferencia de un $20 \%$ de los estudiantes de sexo masculino ${ }^{2,5}$. Por lo anterior, nos parece de interés conocer cuáles son aquellos factores que favorecen o desfavorecen la elección de la cirugía como especialidad.

Antes de proceder a un análisis diferenciado por sexo, es importante señalar que entre los años 1992 y 2002, en los Estados Unidos de Norteamérica se observó una disminución de las postulaciones a los programas de residencia en cirugía general, llegando en el 2002 a quedar vacante el 7\% de las plazas ofrecidas; desde entonces se ha ido revirtiendo parcialmente este hecho, sin llegar a los niveles históricos muy cercanos al 100\%; además, el 9,5\% de las vacantes se completa con estudiantes extranje$\operatorname{ros}^{6}$. Ahora bien, un estudio reciente de Davis EC y cols $^{7}$, ha señalado que en Estados Unidos, a lo largo del período comprendido entre los años 1999 y 2006 la postulación de candidatos de sexo femenino a los programas de cirugía general aumentó de 27\% a $33 \% 7$. Una encuesta realizada en el año 2008 a 2.153 residentes quirúrgicos norteamericanos reveló que hasta un 33\% de los postulantes a un programa de cirugía general son mujeres ${ }^{7}$, pero finalmente, de los residentes en entrenamiento no más del $25 \%$ es de sexo femenino ${ }^{1}$. En un estudio en Chile ${ }^{8}$, se observó 
que el 23,7\% de los residentes de cirugía general eran mujeres, cifra que se compara con el 23,3\% indicado por un estudio en Argentina ${ }^{3}$.

Si tratamos de entender primero la menor demanda actual por las plazas de formación en cirugía general nos encontramos que mayoritariamente la respuesta alude al así llamado “estilo de vida del cirujano"7,9,10. Largas jornadas laborales, horas impredecibles de trabajo, amarre a sistemas de turnos, estrés y dificultades prácticas para equilibrar la vida laboral y profesional, son algunas de las razones que subyacen y han movido a las graduadas de las escuelas de medicina a optar por otras especialidades diferentes a la cirugía ${ }^{11}$. Estudios de reciente publicación confirman que los cirujanos pasan más de 10 horas diarias en el trabajo ${ }^{1}$ y que es mucho más frecuente encontrar mujeres solteras y sin hijos que hombres cirujanos en esa condición ${ }^{2}$. Estos datos, por lo tanto, hablan de que las mujeres dedicadas a la cirugía deben hacer un mayor sacrificio de vida personal para poder ejercer la especialidad tan intensamente como los hombres, y al momento de evaluar la satisfacción laboral de las mujeres cirujanos queda en evidencia que el complemento que desean para poder desarrollar una mayor participación en la especialidad es la posibilidad de contar con más redes de apoyo familiar, de amistad y de comunidades vecinales ${ }^{1}$.

No obstante, considerando todo lo expuesto, y que existe una "cultura quirúrgica” como principal obstáculo para que una mujer sea cirujano, lo concreto es que hoy hay más mujeres protagonistas en el campo quirúrgico ${ }^{9}$. Si bien la elección de la especialidad por parte de un egresado de medicina está determinada por múltiples variables, entre los que se cuenta su personalidad, la relación con residentes en formación durante sus últimos años de medicina y la influencia de modelos a lo largo de sus estudios $^{7,12}$, en Estados Unidos de Norteamérica pudiera influir positivamente en la elección, especialmente para mujeres, la reducción de las horas de trabajo semanal exigibles para un residente de cirugía ${ }^{6}$, que de ser de 100 horas o más, se rebajó a un máximo de 80 horas, según lo estipulado por el Consejo de Acreditación para Graduados de Educación Médica (Accreditation Council on Graduate Medical Education, ACGME) desde el año 2003; en la legislación de la Unión Europea el tope de trabajo semanal para un residente es de 58 horas. La flexibilización del trabajo es, para algunos, elemento determinante al elegir la especialidad ${ }^{5,7}$, como también se ha enfatizado el rol que cumple un mentor en tal decisión, que con su ejemplo es capaz de influir en la percepción que tienen los estudiantes sobre tal especialidad $^{13}$. Esta mayor incorporación de la mujer se da, incluso considerando que una mujer cirujano puede aspirar a un ingreso económico inferior que al de un cirujano hombre ${ }^{1,2,14}$. Junto a esto, hoy son más mujeres cirujanos que participan en el mundo académico y societario ${ }^{3}$. Para lo primero, Davis ${ }^{7}$, encontró un crecimiento del 29\% entre el año 2000 y el 2005. En el mundo de las sociedades científicas también han ocurrido cambios. En los Estados Unidos, en el año 1913 se fundó el Colegio Americano de Cirujanos (American College of Surgeons, ACS) y ese mismo año se hizo socia la primera mujer; para el período 2005-2006 el Colegio fue presidido, por primera vez, por una mujer ${ }^{14}$. En nuestro país, el Capítulo Chileno del ACS fue fundado en 1952 y este año 2011 asumió la Presidencia una de las cuatro mujeres socias. La Sociedad de Cirujanos de Chile, el año 2010, también fue dirigida por una mujer cirujano.

Además de lo referido en cuanto a la elección de la cirugía como especialidad primaria, también se han venido observando diferencias en la elección de una especialidad derivada de la cirugía, hecho que ocurre en más del $75 \%$ de los egresados de un programa de cirugía general en Estados Unidos ${ }^{10}$ y es la aspiración del 73\% de los residentes de cirugía general en nuestro país ${ }^{8}$. Ya se ha remarcado la importancia que tiene el así llamado "estilo de vida quirúrgico” y el rol de un modelo al elegir la cirugía como especialidad ${ }^{7,11}$, a los que se suman otros criterios seguidos por los cirujanos generales para continuar con su especialización; estos hoy se basan en la posibilidad de aumentar sus ingresos, de tener mayor dominio en un área específica y de alejarse de la cirugía general, que demanda mayores esfuerzos $^{6,10}$. Estas últimas razones dan cuenta de la reducción que se observa año tras año de la entrada de cirujanos generales al mundo laboral ${ }^{11}$. Dentro de todos estos factores, ese deseable control de la demanda profesional; la posibilidad de iniciar o mantener una relación estable de pareja y satisfacer aspiraciones de tener hijos son más marcadas en las mujeres, todo lo cual puede englobarse en el concepto de mantener un “estilo de vida” que haga más compatible la vida laboral con la vida familiar",15.

En nuestro medio es escasa la información detallada respecto de las motivaciones y condiciones de los residentes en cirugía general ${ }^{8}$, por lo que creemos que los datos aquí expuestos pueden ser el punto de partida de investigaciones cualitativas que reflejen mejor la mayor incorporación y participación de la mujer en el mundo quirúrgico.

\section{Referencias}

1. Ahmadiyeh N, Cho NL, Kellogg KC, Lipsitz SR, Moore FD Jr, Ashley SW, et al. Career satisfaction of women in 
surgery: perceptions, factors, and strategies. J Am Coll Surg. 2010;210:23-8.

2. Novielli K, Hojat M, Park PK, Gonella JS, Veloski JJ. Change of interest in surgery during medical school: a comparison of men and women. Acad Med. 2001;76:S58-61.

3. Altuna I, Fajre M. El lugar de la mujer en la cirugía Argentina. Rev Argent Cirug. 2006;91:215-25.

4. Espinoza R. Pinceladas de la medicina chilena en el siglo XIX. A 200 años de la Independencia. Rev Chil Cir 2010;62:654-8.

5. Fysh TH, Thomas G, Ellis H. Who wants to be a surgeon? A study of 300 first year medical students. BMC Med Educ. 2007;7:2.

6. Bell RH Jr, Banker MB, Rhodes RS Biester TW, Lewis FR. Graduate medical education in surgery in the United States. Surg Clin North Am. 2007;87:811-23.

7. Davis EC, Risucci DA, Blair PG, Sachdeva AJ. Women in Surgery Residency Programs: Evolving Trends from a National Perspective. J Am Coll Surg. 2011;212:3206.
8. Espinoza R, Danilla S, Valdés F, San Francisco I, Llanos O. Evaluación de los programas de formación de cirugía general. Encuesta a los alumnos de postítulo. Rev Med Chile 2009;137:940-5.

9. Gargiulo DA, Hyman NH, Hebert JC. Women in surgery: do we really understand the deterrents? Arch Surg. 2006;141:405-7.

10. Borman KR, Vick LR, Dattilo JB, Tarpley JL, Mitchell ME. Factors in fellowship selection: effect of services and fellows. J Surg Res. 2009;154:274-8.

11. Bland KI, Isaacs G. Contemporary trends in student selection of medical specialties: the potencial impact of general surgery. Arch Surg. 2002;137:259-67.

12. Townsen CM Jr. Training the surgical scientist: how can that be accomplished? Am Surg. 2007;73:171-3.

13. Bickel J. Women's career development: what does this have to do with men? Ann Surg. 2011;253:644-6.

14. Buyske J. Women in Surgery: the same, yet different. Arch Surg. 2005;140:241-4.

15. Hepp J. Oportunidades para los cirujanos jóvenes: algunas reflexiones. Editorial. Rev Chil Cir 2006;58: 171-2. 